\title{
MULTI-INTERVAL STURM-LIOUVILLE PROBLEMS WITH DISTRIBUTIONAL COEFFICIENTS
}

\author{
ANDRII GORIUNOV \\ Dedicated to Vladimir Mikhailets on the occasion of his 70th birthday anniversary \\ Abstract. The paper investigates spectral properties of multi-interval Sturm- \\ Liouville operators with distributional coefficients. Constructive descriptions of all \\ self-adjoint and maximal dissipative/accumulative extensions and also all generalized \\ resolvents in terms of boundary conditions are given.
}

\section{INTRODUCTION}

Differential operators generated by the Sturm-Liouville expression

$$
l(y)=-\left(p y^{\prime}\right)^{\prime}+q y,
$$

arise in numerous problems of analysis and its applications. The classic assumptions on its coefficients are the following:

$$
q \in C([a, b] ; \mathbb{R}), \quad 0<p \in C^{1}([a, b] ; \mathbb{R}) .
$$

Principal statements of the theory of such operators remain true under more general assumptions that are

$$
q, 1 / p \in L_{1}([a, b], \mathbb{C}) .
$$

However, many problems of mathematical physics require study of differential operators with complex coefficients which are Radon measures or even more singular distributions. In papers $[1,2,3,4]$ a new approach to investigation of such operators was proposed based on definition of these operators as quasi-differential, which allows also to consider differential operators of higher order [5,3].

The purpose of this paper is to develop a spectral theory of Sturm-Liouville operators given on a finite system of bounded intervals under minimal conditions for the regularity of the coefficients.

Multi-interval differential and quasi-differential operators were investigated, particularly, in the papers $[6,7,8,9]$.

Some results of this paper were announced without proof in $[9,10]$.

\section{Preliminary Results}

Let $[a, b]$ be a compact line segment, $m \in \mathbb{N}$, and let $a=a_{0}<a_{1}<\cdots<a_{m}=b$ be a partition of $[a, b]$ into $m$ parts. Let us consider the space $L_{2}([a, b], \mathbb{C})$ as a direct sum $\oplus_{k=1}^{m} L_{2}\left(\left[a_{k-1}, a_{k}\right], \mathbb{C}\right)$ which consists of vector functions $f=\oplus_{k=1}^{m} f_{k}$ such that $f_{k} \in L_{2}\left(\left[a_{k-1}, a_{k}\right], \mathbb{C}\right)$.

2010 Mathematics Subject Classification. 34L40, 34B45.

Key words and phrases. Sturm-Liouville operator; multi-interval boundary value problem; distributional coefficients; self-adjoint extension; maximal dissipative extension. 
Let on every interval $\left(a_{k-1}, a_{k}\right), k \in\{1, \ldots, m\}$ the formal Sturm-Liouville differential expression

$$
l_{k}(y)=-\left(p_{k}(t) y^{\prime}\right)^{\prime}+q_{k}(t) y+i\left(\left(r_{k}(t) y\right)^{\prime}+r_{k}(t) y^{\prime}\right),
$$

be given with the coefficients $p_{k}, q_{k}$, and $r_{k}$ satisfying the conditions

$$
q_{k}=Q_{k}^{\prime}, \quad \frac{1}{\sqrt{\left|p_{k}\right|}}, \frac{Q_{k}}{\sqrt{\left|p_{k}\right|}}, \frac{r_{k}}{\sqrt{\left|p_{k}\right|}} \in L_{2}\left(\left[a_{k-1}, a_{k}\right], \mathbb{C}\right),
$$

where the derivatives $Q_{k}^{\prime}$ are understood in the sense of distributions.

Similarly to [3] (see also [1, 4]), using the coefficients of the expression (1) we introduce the quasi-derivatives on every segment $\left[a_{k-1}, a_{k}\right]$ in the following way:

$$
\begin{aligned}
D_{k}^{[0]} y & :=y \\
D_{k}^{[1]} y & :=p_{k} y^{\prime}-\left(Q_{k}+i r_{k}\right) y \\
D_{k}^{[2]} y & :=\left(D_{k}^{[1]} y\right)^{\prime}+\frac{Q_{k}-i r_{k}}{p_{k}} D_{k}^{[1]} y+\frac{Q_{k}^{2}+r_{k}^{2}}{p_{k}} y .
\end{aligned}
$$

Also denote

$$
\widehat{y}_{k}(t)=\left(D_{k}^{[0]} y(t), D_{k}^{[1]} y(t)\right) \in \mathbb{C}^{2}
$$

for all $t \in\left[a_{k-1}, a_{k}\right]$. Under assumptions (2) these expressions are Shin-Zettl quasiderivatives (see $[12,11]$ ). One can easily verify that for the smooth coefficients $p_{k}, q_{k}$ and $r_{k}$ the equality $l_{k}(y)=-D_{k}^{[2]} y$ holds.

Therefore one may correctly define expressions (1) under assumptions (2) as Shin-Zettl quasi-differential expressions,

$$
l_{k}[y]:=-D_{k}^{[2]} y .
$$

The corresponding Shin-Zettl matrices (see $[11,12])$ have the form

$$
A_{k}=\left(\begin{array}{cc}
\frac{Q+i r}{p} & \frac{1}{p} \\
-\frac{Q^{2}+r^{2}}{p} & -\frac{Q-i r}{p}
\end{array}\right) \in L_{1}\left([a, b] ; \mathbb{C}^{2 \times 2}\right) .
$$

Then on the Hilbert spaces $L_{2}\left(\left(a_{k-1}, a_{k}\right), \mathbb{C}\right)$ minimal and maximal differential operators are defined, which are generated by the quasi-differential expressions $l_{k}[y]$ (see $[12,11])$

$$
\begin{gathered}
L_{k, 1}: y \rightarrow l_{k}[y], \quad \operatorname{Dom}\left(L_{k, 1}\right):=\left\{y \in L_{2} \mid y, D_{k}^{[1]} y \in A C\left(\left[a_{k-1}, a_{k}\right], \mathbb{C}\right), D_{k}^{[2]} y \in L_{2}\right\}, \\
L_{k, 0}: y \rightarrow l_{k}[y], \quad \operatorname{Dom}\left(L_{k, 0}\right):=\left\{y \in \operatorname{Dom}\left(L_{k, 1}\right) \mid \widehat{y}_{k}\left(a_{k-1}\right)=\widehat{y}_{k}\left(a_{k}\right)=0 .\right\} .
\end{gathered}
$$

Results of $[11,12]$ for general Shin-Zettl quasi-differential operators together with formula (3) imply that the operators $L_{k, 1}, L_{k, 0}$ are closed and densely defined on the space $L_{2}\left(\left[a_{k-1}, a_{k}\right], \mathbb{C}\right)$.

In the case where $p_{k}, q_{k}$ and $r_{k}$ are real-valued, the operator $L_{k, 0}$ is symmetric with the deficiency index $(2,2)$ and

$$
L_{k, 0}^{*}=L_{k, 1}, \quad L_{k, 1}^{*}=L_{k, 0} .
$$

\section{Boundary-Value problems. Symmetric case}

We consider the space $L_{2}([a, b], \mathbb{C})$ as a direct sum $\oplus_{k=1}^{m} L_{2}\left(\left[a_{k-1}, a_{k}\right], \mathbb{C}\right)$ which consists of vector functions $f=\oplus_{k=1}^{m} f_{k}$ such that $f_{k} \in L_{2}\left(\left[a_{k-1}, a_{k}\right], \mathbb{C}\right)$. In this space $L_{2}([a, b], \mathbb{C})$ we consider the maximal and the minimal operators $L_{\max }=\oplus_{k=1}^{m} L_{k, 1}$ and $L_{\min }=\oplus_{k=1}^{m} L_{k, 0}$.

It is easy to see that the operators $L_{\max }, L_{\min }$ are closed and densely defined on the space $L_{2}([a, b], \mathbb{C})$. 
Throughout the rest of the paper we assume the functions $p_{k}, q_{k}$, and $r_{k}$ to be realvalued for all $k$ and therefore the operators $L_{k, 0}$ are symmetric with the deficiency indices $(2,2)$. Then the operator $L_{\min }$ is symmetric with the deficiency index $(2 m, 2 m)$ and

$$
L_{\min }^{*}=L_{\max }, \quad L_{\max }^{*}=L_{\min } .
$$

Then naturally arises the problem of describing all its self-adjoint, maximal dissipative, and maximal accumulative extensions in terms of homogeneous boundary conditions of the canonical form. For this purpose it is convenient to apply the approach based on the concept of boundary triplets. It was developed in the papers by Kochubei [13], see also the monograph [14] and references therein.

Note that the minimal operator $L_{\min }$ can not be semi-bounded even in the case of a single-interval boundary-value problem since the function $p$ can change the sign.

Recall that a boundary triplet of a closed densely defined symmetric operator $T$ with equal (finite or infinite) deficiency indices is a triplet $\left(H, \Gamma_{1}, \Gamma_{2}\right)$ where $H$ is an auxiliary Hilbert space and $\Gamma_{1}, \Gamma_{2}$ are linear maps from $\operatorname{Dom}\left(T^{*}\right)$ into $H$ such that

(1) for any $f, g \in \operatorname{Dom}\left(T^{*}\right)$ there holds

$$
\left(T^{*} f, g\right)_{\mathcal{H}}-\left(f, T^{*} g\right)_{\mathcal{H}}=\left(\Gamma_{1} f, \Gamma_{2} g\right)_{H}-\left(\Gamma_{2} f, \Gamma_{1} g\right)_{H},
$$

(2) for any $g_{1}, g_{2} \in H$ there is a vector $f \in \operatorname{Dom}\left(T^{*}\right)$ such that $\Gamma_{1} f=g_{1}$ and $\Gamma_{2} f=g_{2}$.

The definition of the boundary triplet implies that $f \in \operatorname{Dom}(T)$ if and only if $\Gamma_{1} f=$ $\Gamma_{2} f=0$. A boundary triplet $\left(H, \Gamma_{1}, \Gamma_{2}\right)$ with $\operatorname{dim} H=n$ exists for any symmetric operator $T$ with equal non-zero deficiency indices $(n, n)(n \leq \infty)$, but it is not unique.

For the minimal quasi-differential operators $L_{k, 0}$ the boundary triplet is explicitly given by the following theorem which follows from the results of [2].

Theorem 1. For every $k=1, \ldots, m$ the triplet $\left(\mathbb{C}^{2}, \Gamma_{1, k}, \Gamma_{2, k}\right)$, where $\Gamma_{1, k}, \Gamma_{2, k}$ are linear maps,

$$
\Gamma_{1, k} y:=\left(D_{k}^{[1]} y\left(a_{k-1}+\right),-D_{k}^{[1]} y\left(a_{k}-\right)\right), \Gamma_{2, k} y:=\left(y\left(a_{k-1}+\right), y\left(a_{k}-\right)\right),
$$

from $\operatorname{Dom}\left(L_{k, 1}\right)$ onto $\mathbb{C}^{2}$ is a boundary triplet for the operator $L_{k, 0}$.

For the minimal operator $L_{\min }$ in the space $L_{2}([a, b], \mathbb{C})$ the boundary triplet is explicitly given by the following theorem.

Theorem 2. The triplet $\left(\mathbb{C}^{2 m}, \Gamma_{1}, \Gamma_{2}\right)$, where $\Gamma_{1}, \Gamma_{2}$ are linear maps,

$$
\Gamma_{1} y:=\left(\Gamma_{1,1} y, \Gamma_{1,2} y, \ldots, \Gamma_{1, m} y\right), \Gamma_{2} y:=\left(\Gamma_{2,1} y, \Gamma_{2,2} y, \ldots, \Gamma_{2, m} y\right),
$$

from $\operatorname{Dom}\left(L_{\max }\right)$ onto $\mathbb{C}^{2 m}$ is a boundary triplet for the operator $L_{\text {min }}$.

Proof. We need to verify that both conditions of the definition of the boundary triplet are fulfilled.

But since for every $k=1, \ldots, m\left(\mathbb{C}^{2}, \Gamma_{1, k}, \Gamma_{2, k}\right)$ is a boundary triplet for the operator $L_{k, 0}$, then we have the following.

1) Let $y=\oplus_{k=1}^{m} y_{m}, z=\oplus_{k=1}^{m} z_{m}$ be two arbitrary functions which belong to $\operatorname{Dom}\left(L_{\max }\right)$. Then

$$
\begin{aligned}
\left(L_{\max } y, z\right)-\left(y, L_{\max } z\right) & =\sum_{k=1}^{m}\left(L_{k, 1} y_{k}, z_{k}\right)-\sum_{k=1}^{m}\left(y_{k}, L_{k, 1} z_{k}\right)= \\
& =\sum_{k=1}^{m}\left(\Gamma_{1, k} y_{k}, \Gamma_{2, k} z_{k}\right)-\sum_{k=1}^{m}\left(\Gamma_{2, k} y_{k}, \Gamma_{1, k} z_{k}\right)= \\
& =\left(\Gamma_{1} y, \Gamma_{2} z\right)-\left(\Gamma_{2} y, \Gamma_{1} z\right) .
\end{aligned}
$$


2) Let $g_{1}, g_{2} \in \mathbb{C}^{2 m}$, and therefore $g_{1}=\left(g_{1,1}, \ldots, g_{1, m}\right), g_{2}=\left(g_{2,1}, \ldots, g_{2, m}\right)$, where $g_{i k} \in \mathbb{C}^{2}$. Then for all $k=1, \ldots, m$ there are functions $y_{k} \in \operatorname{Dom}\left(L_{k, 1}\right.$ such that $\Gamma_{1, k} y_{k}=g_{1, k}, \Gamma_{2, k} y_{k}=g_{2, k}$, which implies that the definition of boundary triplet is fulfilled.

Denote by $L_{K}$ the restriction of the operator $L_{\max }$ onto the set of functions $y \in \operatorname{Dom}\left(L_{\max }\right)$ satisfying the homogeneous boundary condition

$$
(K-I) \Gamma_{1} y+i(K+I) \Gamma_{2} y=0,
$$

where $K$ is an arbitrary bounded operator on the space $\mathbb{C}^{2 m}$.

Similarly, denote by $L^{K}$ the restriction of $L_{\max }$ onto the set of functions $y \in \operatorname{Dom}\left(L_{\max }\right)$ satisfying the homogeneous boundary condition

$$
(K-I) \Gamma_{1} y-i(K+I) \Gamma_{2} y=0,
$$

where $K$ is an arbitrary bounded operator on the space $\mathbb{C}^{2 m}$.

Theorem 1 leads to the following description of all self-adjoint, maximal dissipative, and maximal accumulative extensions of the operator $L_{\max }$.

Theorem 3. Every $L_{K}$ with $K$ being a contracting operator in the space $\mathbb{C}^{2 m}$, is a maximal dissipative extension of the operator $L_{\text {min }}$. Similarly every $L^{K}$ with $K$ being a contracting operator in $\mathbb{C}^{2 m}$, is a maximal accumulative extension of the operator $L_{\text {min }}$.

Conversely, for any maximal dissipative (respectively, maximal accumulative) extension $\widetilde{L}$ of the operator $L_{\text {min }}$ there exists a unique contracting operator $K$ such that $\widetilde{L}=L_{K} \quad$ (respectively, $\left.\widetilde{L}=L^{K}\right)$.

The extensions $L_{K}$ and $L^{K}$ are self-adjoint if and only if $K$ is a unitary operator on $\mathbb{C}^{2 m}$.

The mappings $K \rightarrow L_{K}$ and $K \rightarrow L^{K}$ are injective.

Proof. The assertion of the theorem follows from Theorem 1 together with [14, Th. 1.6] for boundary triplets of a general symmetric operator.

\section{REAL EXTEnsions AND LOCAL BOUNDARY CONDITIONS} if

Recall that a linear operator $T$ acting on the Hilbert space $L_{2}([a, b], \mathbb{C})$ is called real

(1) for every function $f$ from $\operatorname{Dom}(T)$ the complex conjugate function $\bar{f}$ also lies in $\operatorname{Dom}(T)$

(2) the operator $T$ maps complex conjugate functions into complex conjugate functions, that is, $T(\bar{f})=\overline{T(f)}$.

Theorem 4. Suppose that all the coefficients $r_{k} \equiv 0, k \in\{1, \ldots, m\}$. Then the maximal and the minimal quasi-differential operators $L_{\max }$ and $L_{\min }$ are real.

All real maximal dissipative and maximal accumulative extensions of the real symmetric quasi-differential operator $L_{\text {min }}$ are self-adjoint.

The self-adjoint extensions $L_{K}$ or $L^{K}$ are real if and only if the unitary matrix $K$ is symmetric.

Proof. As we assumed at the beginning of this section, all functions $p_{k}, Q_{k} \in L_{2}\left(\left[a_{k-1}, a_{k}\right], \mathbb{R}\right)$. As for any $k$ the coefficients of the quasi-derivatives are real-valued functions, one has

$$
D_{k}^{[1]} \bar{y}=\overline{D_{k}^{[1]} y}, \quad k=1, \ldots, m,
$$

which implies that $l_{k}(\bar{y})=\overline{l_{k}(y)}$. Therefore for any $y \in \operatorname{Dom}\left(L_{k, 1}\right)$ we have $\bar{y} \in$ $\operatorname{Dom}\left(L_{k, 1}\right)$ and $L_{k, 1}(\bar{y})=\overline{L_{k, 1}(y)}$ and for any $y \in \operatorname{Dom}\left(L_{k, 0}\right)$ we have $\bar{y} \in \operatorname{Dom}\left(L_{k, 0}\right)$.

Thus the assertion of the theorem yields that $L_{k, 1}$ and $L_{k, 0}$ are real. Therefore the operators $L_{\max }$ and $L_{\min }$ are real being the direct sums. 
Let now $L_{K}$ be a real maximal dissipative extension of $L_{\text {min }}$. Again, one easily sees that $\Gamma_{1} \bar{y}=\overline{\Gamma_{1} y}$ and $\Gamma_{2} \bar{y}=\overline{\Gamma_{2} y}$. Then for an arbitrary function $y \in \operatorname{Dom}\left(L_{K}\right)$ we have

$$
(K-I) \Gamma_{1} \bar{y}+i(K+I) \Gamma_{2} \bar{y}=0,
$$

therefore

$$
(\bar{K}-I) \Gamma_{1} y-i(\bar{K}+I) \Gamma_{2} y=0,
$$

so, in other words, $L_{K} \subset L^{\bar{K}}$. Thus the maximal dissipative extension $L_{K}$ is also accumulative, therefore, the operator $L_{K}$ is self-adjoint and we obtain $L_{K}=L^{\bar{K}}$.

Due to [2, Remark 4.3] we have that $L_{K}=L^{\bar{K}}$ if and only if $K^{-1}=\bar{K}$. Since $K$ is a unitary matrix, $K^{-1}=\overline{K^{T}}$, which gives $K=K^{T}$.

Similarly one can show that the maximal accumulative extension $L^{K}$ is real if and only if it is self-adjoint and $K=K^{T}$.

All functions from $\operatorname{Dom}\left(L_{\max }\right)$, together with their first quasi-derivatives, belong to $\oplus_{k=1}^{m} A C\left(\left[a_{k-1}, a_{k}\right], \mathbb{C}\right)$. This implies that the following definition is correct.

Denote by $[f]_{t-}$ the left germ and by $[f]_{t+}$ the right germ of the continuous function $f$ at a point $t$. Similarly to the paper [2] we say that boundary conditions that define the operator $L \subset L_{\max }$ are called local if, for any functions $y \in \operatorname{Dom}(L)$ and for any $y_{1}, \ldots, y_{m} \in \operatorname{Dom}\left(L_{\max }\right)$, the identities

$$
\left[y_{j}\right]_{a_{j}-}=[y]_{a_{j}-}, \quad\left[y_{j}\right]_{a_{j}+}=[y]_{a_{j}+}, \quad\left[y_{j}\right]_{a_{k}-}=\left[y_{j}\right]_{a_{k}+}=0, \quad k \neq j
$$

imply that $y_{j} \in \operatorname{Dom}(L)$. For $j=0$ and $j=m$ we consider only the right and left germs, respectively.

The following statement gives a description of the extensions $L_{K}$ and $L^{K}$ which are given by local boundary conditions.

Theorem 5. The boundary conditions (5) and (6) defining the extensions $L_{K}$ and $L^{K}$, respectively, are local if and only if the matrix $K$ has the block form

$$
K=\left(\begin{array}{cccc}
K_{a_{0}} & 0 & \ldots & 0 \\
0 & K_{a_{1}} & \ldots & 0 \\
0 & 0 & \ldots & K_{a_{n}}
\end{array}\right),
$$

where $K_{a_{1}}$ and $K_{a_{n}} \in \mathbb{C}$ and other $K_{a_{j}} \in \mathbb{C}^{2 \times 2}$.

Proof. Let us consider the extension $L_{K}$ defined by the boundary conditions (5).

Note that for all $f, g \in \operatorname{Dom}\left(L_{\max }\right)$ the identity $[f]_{a_{k}-}=[g]_{a_{k}-}$ implies that

$$
\left[D_{k}^{[1]} f\right]_{a_{k}-}=\left[D_{k}^{[1]} g\right]_{a_{k}-}, \quad k=1, \ldots, m,
$$

and therefore

$$
D_{k}^{[1]} f\left(a_{k}-\right)=D_{k}^{[1]} g\left(a_{k}-\right), \quad f\left(a_{k}-\right)=g\left(a_{k}-\right) .
$$

Similarly, the identity $[f]_{a_{k}+}=[g]_{a_{k}+}$ implies that

$$
\left[D_{k+1}^{[1]} f\right]_{a_{k}+}=\left[D_{k+1}^{[1]} g\right]_{a_{k}+}, \quad k=0, \ldots, m-1,
$$

and therefore

$$
D_{k+1}^{[1]} f\left(a_{k}+\right)=D_{k+1}^{[1]} g\left(a_{k}+\right), \quad f\left(a_{k}+\right)=g\left(a_{k}+\right) .
$$


If $K$ has the form (7), then the boundary conditions (5) can be written in the form of the system

$$
\left\{\begin{array}{r}
\left(K_{a_{0}}-1\right) D_{1}^{[1]} y\left(a_{0}+\right)+i\left(K_{a_{0}}+1\right) y\left(a_{0}+\right)=0 \\
\left(K_{a_{1}}-I\right)\left(\begin{array}{c}
-D_{1}^{[1]} y\left(a_{1}-\right) \\
D_{2}^{[1]} y\left(a_{1}+\right)
\end{array}\right)+i\left(K_{a_{1}}+I\right)\left(\begin{array}{l}
y\left(a_{1}-\right) \\
y\left(a_{1}+\right)
\end{array}\right)=0 \\
\ldots \\
\left(K_{a_{m}}-1\right)\left(-D_{m}^{[1]} y\left(a_{m}-\right)\right)+i\left(K_{a_{m}}+1\right) y\left(a_{m}-\right)=0 .
\end{array}\right.
$$

It is evident that these boundary conditions are local.

Conversely, suppose that the boundary conditions (5) are local.

Denote the elements of the matrix $K$ by

$$
K=\left(\begin{array}{cccc}
k_{1,1} & k_{1,2} & \ldots & k_{1,2 m} \\
k_{2,1} & k_{2,2} & \ldots & k_{2,2 m} \\
\ldots & \ldots & \ldots & \ldots \\
k_{2 m, 1} & k_{2 m, 2} & \ldots & k_{2 m, 2 m}
\end{array}\right),
$$

where $k_{r, j} \in \mathbb{C}$. We need to prove that $k_{r j}=0$ except for the following cases: $r=j=1$; $r=j=2 m ; r=2 p+1, j \in\{r, r+1\}, p=0, \ldots, m-1 ; r=2 p, j \in\{r-1, r\}$, $p=1, \ldots, m-1$.

Boundary conditions (5) then take the form of $2 m$ equations,

$$
\left\{\begin{array}{c}
\sum_{j=1}^{m}\left(k_{2 p+1,2 j-1} D_{j}^{[1]} y\left(a_{j-1}+\right)-k_{2 p+1,2 j} D_{j}^{[1]} y\left(a_{j}-\right)\right)-D_{p+1}^{[1]} y\left(a_{p}+\right)+ \\
+i \sum_{j=1}^{m}\left(k_{2 p+1,2 j-1} y\left(a_{j-1}+\right)+k_{2 p+1,2 j} y\left(a_{j}-\right)\right)+i y\left(a_{p}+\right)=0, \\
r=2 p+1, p=0, \ldots, m-1, \\
\sum_{j=1}^{m}\left(k_{2 p, 2 j-1} D_{j}^{[1]} y\left(a_{j-1}+\right)-k_{2 p, 2 j} D_{j}^{[1]} y\left(a_{j}-\right)\right)+D_{p}^{[1]} y\left(a_{p}-\right)+ \\
+i \sum_{j=1}^{m}\left(k_{2 p, 2 j-1} y\left(a_{j-1}+\right)+K_{2 p, 2 j} y\left(a_{j}-\right)\right)+i y\left(a_{p}-\right)=0 \\
r=2 p, p=1, \ldots, m
\end{array}\right.
$$

The definition of local boundary conditions implies that any function $y_{s}$ such that $\left[y_{s}\right]_{a_{s}-}=[y]_{a_{s}-},\left[y_{s}\right]_{a_{s}+}=[y]_{a_{s}+}$, and $\left[y_{s}\right]_{a_{k}-}=[y]_{a_{k}+}=0, k \neq s$, must also satisfy this system. Therefore we have

$$
k_{r, 2 s+1} D_{s+1}^{[1]} y\left(a_{s}+\right)-k_{r, 2 s} D_{s}^{[1]} y\left(a_{s}-\right)+i k_{r, 2 s+1} y\left(a_{s}+\right)+i k_{r, 2 s} y\left(a_{s}-\right)=0,
$$

or

$$
k_{r, 2 s+1}\left(D_{s+1}^{[1]} y\left(a_{s}+\right)+i y\left(a_{s}+\right)\right)+k_{r, 2 s}\left(-D_{s}^{[1]} y\left(a_{s}-\right)+i y\left(a_{s}-\right)\right)=0,
$$

where $r=1, \ldots, 2 m$, and $s$ runs from 1 to $n-1, s \neq p$, where $r=2 p$ or $r=2 p+1$. Therefore these equations contain all $k_{r s}$ we need to prove to be equal to 0 .

Let us return to boundary conditions (5) and rewrite them in a parametric form. For any vector $F=\left(F_{1}, \ldots, F_{2 m}\right) \in \mathbb{C}^{2 m}$, we consider the vectors $-i(K+I) F$ and $(K-I) F$. Due to the definition of a boundary triplet the is a function $y_{F} \in \operatorname{Dom}\left(L_{\max }\right)$ such that

$$
\left\{\begin{aligned}
-i(K+I) F & =\Gamma_{1} y_{F}, \\
(K-I) F & =\Gamma_{2} y_{F} .
\end{aligned}\right.
$$


One easily sees that $y_{F}$ satisfies boundary conditions (5) and therefore $y_{F} \in \operatorname{Dom}\left(L_{K}\right)$. We rewrite the last system in the form

$$
\left\{\begin{array}{l}
-i \sum_{j=1}^{2 m} k_{r, j} F_{j}-i F_{r}=-D_{p}^{[1]} y_{F}\left(a_{p}-\right), \quad r=2 p+1, p=0, \ldots, n, \\
-i \sum_{j=1}^{2 m} k_{r, j} F_{j}-i F_{r}=D_{p+1}^{[1]} y_{F}\left(a_{p}+\right), \quad r=2 p, p=1, \ldots, n, \\
-i \sum_{j=1}^{2 m} k_{r, j} F_{j}-F_{r}=y_{F}\left(a_{p}-\right), \quad r=2 p+1, p=0, \ldots, n, \\
-i \sum_{j=1}^{2 m} k_{r, j} F_{j}-F_{r}=y_{F}\left(a_{p}+\right), \quad r=2 p, p=1, \ldots, n,
\end{array}\right.
$$

which results in $-D_{p}^{[1]} y_{F}\left(a_{p}-\right)+i y_{F}\left(a_{p}-\right)=-2 i F_{2 p}$ and $D_{p+1}^{[1]} y_{F}\left(a_{p}+\right)+i y_{F}\left(a_{p}+\right)=$ $-2 i F_{2 p+1}$ for arbitrary numbers $F_{j} \in \mathbb{C}$.

Substituting this into (8) we have that

$$
k_{r, 2 s+1} F_{2 s+1}+k_{r, 2 s} i F_{2 s}=0
$$

for arbitrary numbers $F_{j}$, therefore obviously $k_{r, 2 s+1}=k_{r, 2 s}=0$ and the Theorem is proved.

\section{Generalized RESOlvents}

Let us recall that a generalized resolvent of a closed symmetric operator $L$ on a Hilbert space $\mathcal{H}$ is an operator-valued function $\lambda \mapsto R_{\lambda}$, defined on $\mathbb{C} \backslash \mathbb{R}$ which can be represented as

$$
R_{\lambda} f=P^{+}\left(L^{+}-\lambda I^{+}\right)^{-1} f, \quad f \in \mathcal{H},
$$

where $L^{+}$is a self-adjoint extension of the operator $L$ that acts on a certain Hilbert space $\mathcal{H}^{+} \supset \mathcal{H}, I^{+}$is the identity operator on $\mathcal{H}^{+}$, and $P^{+}$is the orthogonal projection operator from $\mathcal{H}^{+}$onto $\mathcal{H}$. It is known that an operator-valued function $R_{\lambda}$ is a generalized resolvent of a symmetric operator $L$ if and only if it can be represented as

$$
\left(R_{\lambda} f, g\right)_{\mathcal{H}}=\int_{-\infty}^{+\infty} \frac{d\left(F_{\mu} f, g\right)}{\mu-\lambda}, \quad f, g \in \mathcal{H}
$$

where $F_{\mu}$ is a generalized spectral function of the operator $L$. This implies that the operator-valued function $F_{\mu}$ has the following properties.

(1) For $\mu_{2}>\mu_{1}$ the difference $F_{\mu_{2}}-F_{\mu_{1}}$ is a bounded non-negative operator.

(2) $F_{\mu+}=F_{\mu}$ for any real $\mu$.

(3) For any $x \in \mathcal{H}$ the following identities hold:

$$
\lim _{\mu \rightarrow-\infty}\left\|F_{\mu} x\right\|_{\mathcal{H}}=0, \quad \lim _{\mu \rightarrow+\infty}\left\|F_{\mu} x-x\right\|_{\mathcal{H}}=0 .
$$

The following theorem provides a parametric description of all generalized resolvents of the symmetric operator $L_{\min }$ (see also [15]).

Theorem 6. 1) Every generalized resolvent $R_{\lambda}$ of the operator $L_{\min }$ in the half-plane $\operatorname{Im} \lambda<0$ acts by the rule $R_{\lambda} h=y$, where $y$ is a solution of the boundary-value problem

$$
\begin{gathered}
l(y)=\lambda y+h, \\
(K(\lambda)-I) \Gamma_{1} f+i(K(\lambda)+I) \Gamma_{2} f=0 .
\end{gathered}
$$

Here $h(x) \in L_{2}([a, b], \mathbb{C})$ and $K(\lambda)$ is a $2 m \times 2 m$ matrix-valued function which is holomorphic in the lower half-plane and such that $\|K(\lambda)\| \leq 1$. 
2) In the half-plane $\operatorname{Im} \lambda>0$ every generalized resolvent of the operator $L_{\text {min }}$ acts by $R_{\lambda} h=y$, where $y$ is a solution of the boundary-value problem

$$
\begin{gathered}
l(y)=\lambda y+h, \\
(K(\lambda)-I) \Gamma_{1} f-i(K(\lambda)+I) \Gamma_{2} f=0 .
\end{gathered}
$$

Here $h(x) \in L_{2}([a, b], \mathbb{C})$ and $K(\lambda)$ is a $2 m \times 2 m$ matrix-valued function which is holomorphic in the upper half-plane and satisfies $\|K(\lambda)\| \leq 1$.

This parametrization of the generalized resolvents by the matrix-valued functions $K(\lambda)$ is bijective.

Proof. The Theorem is just an application of Theorem 2 and [15, Theorem 1 and Remark 1] which proves the description of generalized resolvents in terms of boundary triplets. Namely, one requires to take as an auxiliary Hilbert space $\mathbb{C}^{m}$ and as the operator $\gamma y:=\left\{\Gamma_{1} y, \Gamma_{2} y\right\}$.

Acknowledgement. The authors have received funding from the European Union's Horizon 2020 research and innovation program under the Marie Skłodowska-Curie grant agreement No 873071.

\section{REFERENCES}

1. A. S. Goriunov, V. A. Mikhailets, Regularization of singular Sturm-Liouville equations, Meth. Funct. Anal. Topol. 16 (2010), no. 2, 120-130.

2. A. S. Goriunov, V. A. Mikhailets, K. Pankrashkin, Formally self-adjoint quasi-differential operators and boundary-value problems, Electron. J. Diff. Equ. 2013 (2013), no. 101, 1-16.

3. K. A. Mirzoev, A. A. Shkalikov, Differential operators of even order with distribution coeffcients, Math. Notes. 99 (2016), no. 5, 779-784.

4. J. Eckhardt, F. Gesztesy, R. Nichols, G. Teschl, Weyl-Titchmarsh theory for Sturm-Liouville operators with distributional coefficients, Opuscula Mathematica. 33 (2013), no. 3, 467-563.

5. A. S. Goriunov, V. A. Mikhailets, Regularization of two-term differential equations with singular coefficients by quasiderivatives, Ukrainian Math. J. 63 (2012), no. 9, 1361-1378.

6. W. N. Everitt, A. Zettl, Sturm-Liouville differential operators in direct sum spaces, Rocky Mountain J. Math. 16 (1986), no. 3, 497-516.

7. W. N. Everitt, A. Zettl, Quasi-differential operators generated by a countable number of expressions on the real line, Proc. London Math. Soc. 64 (1992), no. 3, 524-544.

8. M. S. Sokolov, Representation results for operators generated by a quasi-differential multiinterval system in a Hilbert direct sum space, Rocky Mountain J. Math. 36 (2006), no. 2, 721-739.

9. A. S. Goriunov, Multi-interval Sturm-Liouville boundary-value problems with distributional potentials, Dopov. Nats. Acad. Nauk. Ukr. (2014), no. 7, 43-47.

10. A. S. Goriunov, Multi-interval dissipative Sturm-Liouville boundary-value problems with distributional coefficients, Dopov. Nats. Acad. Nauk. Ukr. (2020), no. 7, to appear.

11. A. Zettl, Formally self-adjoint quasi-differential operators, Rocky Mountain J. Math. 5 (1975), no. 3, 453-474.

12. W. N. Everitt, L. Markus, Boundary value problems and symplectic algebra for ordinary differential and quasi-differential operators, American Mathematical Society, Providence, 1999.

13. A. N. Kochubei, Extensions of symmetric operators and of symmetric binary relations, Math. Notes 17 (1975), no. 1, 25-28.

14. V. I. Gorbachuk, M. L. Gorbachuk, Boundary value problems for operator differential equations. Translated and revised from the 1984 Russian original., Mathematics and its Applications (Soviet Series), 48, Kluwer, Dordrecht, 1991.

15. V. M. Bruk, A certain class of boundary value problems with a spectral parameter in the boundary condition. (Russian), Mat. Sb. (N.S.) 100(142) (1976), no. 2(6), 210-216.

Institute of Mathematics of National Academy of Sciences of Ukraine, Kyiv, Ukraine

E-mail address: goriunov@imath.kiev.ua

Received 09.06.2020 